\title{
Intertrial priming due to distractor repetition is eliminated in homogeneous contexts
}

\author{
Tobias Feldmann-Wüstefeld ${ }^{1,2}$ • Anna Schubö ${ }^{1}$
}

Published online: 10 May 2016

(C) The Psychonomic Society, Inc. 2016

\begin{abstract}
Targets are found more easily in a visual search task when their feature is repeatedly presented, an effect known as intertrial priming. Recent findings suggest that priming of distractors can also improve search performance by facilitated suppression of repeated distractor features. The efficacy of intertrial priming for targets can be potentiated by the expectancy of a specific target feature; systematic repetition shows larger intertrial priming than random repetition. For distractors, the underlying mechanism is less clear. We used the systematic lateralization approach to disentangle target- and distractorrelated processing with subcomponents of the N2pc. We found no modulation of the $\mathrm{N}_{\mathrm{T}}$ component, which reflects prioritization of target processing. The $\mathrm{N}_{\mathrm{D}}$ component, which reflects attentional capture by irrelevant stimuli, however, showed intertrial priming: $\mathrm{N}_{\mathrm{D}}$ monotonically decreased with repetition of a distractor color, but only if a specific distractor feature was expected, and if the context induced a search that was vulnerable to attentional capture. These observations suggest that distractor priming only improves visual search if volitional control is relatively high. The results also suggest that intertrial priming for distractors is due to decreased attentional capture by repeatedly presented distractors, whereas target processing remains unaffected.
\end{abstract}

Tobias Feldmann-Wüstefeld

tobiasfw@uchicago.edu

1 Experimental and Biological Psychology, University of Marburg, Marburg, Germany

2 Department of Psychology, University of Chicago, Chicago, USA
Keywords Visual attention - Intertrial priming $\cdot$ Priming of pop-out $\cdot$ Attentional capture $\cdot \mathrm{N} 2 \mathrm{pc} \cdot \mathrm{P}_{\mathrm{D}} \cdot \mathrm{N}_{\mathrm{T}} \cdot \mathrm{N}_{\mathrm{D}}$

To cope with the vast amount of incoming information, the visual system selects stimuli based not only on intentions of the observer and physical salience but also on recent selection history. For example, RTs for targets decrease when a target feature is identical to a preceding trial. This intertrial priming effect was originally found for pop-out targets that are defined by a unique feature that clearly separates them from all visual background and termed "priming of pop-out" (Becker, Ansorge, \& Horstmann, 2009; Eimer, Kiss, \& Cheung, 2010; Found \& Müller, 1996; Kristjánsson, Vuilleumier, Schwartz, MacAluso, \& Driver, 2007; Lamy, Antebi, Aviani, \& Carmel, 2008; Lamy, Carmel, Egeth, \& Leber, 2006; Maljkovic \& Nakayama, 1994). Intertrial priming has been found for various stimulus dimensions such as color, spatial frequency, orientation, and shape (Kristjánsson \& Campana, 2010). Since intertrial priming occurred in search tasks that controlled for physical salience, and also ocurred when observers were informed about the target feature in the upcoming trial (Becker, 2008; Folk \& Remington, 2008; Maljkovic \& Nakayama, 1994), it was argued that intertrial priming is independent of bottom-up and top-down processing (Wang, Kristjánsson, \& Nakayama, 2005) and may be a good example for a third factor, "selection history" (Awh, Belopolsky, \& Theeuwes, 2012; Leber \& Egeth, 2006).

Most studies investigating intertrial priming cannot dissociate the relative contribution of target prioritization and distractor suppression to the facilitatory effect of feature repetition; in feature change trials, target and distractor features are usually swapped. That is, trials in which a target feature was repeated were also trials in which a distractor color was repeated. Analogously, trials in which a target feature changed 
were also trials in which a distractor color changed. Accordingly, any facilitatory effect of target feature repetition was equivalent to a facilitatory effect of distractor feature repetition. Most authors, interestingly, attributed the facilitatory effect to target repetition rather than to distractor suppression, even though distractor suppression may play a more important role under some circumstances (Geyer, Müller, \& Krummenacher, 2006; Lamy, Yashar, \& Ruderman, 2013). In one experiment of the original study by Maljkovic and Nakayama (1994, Experiment 8), target color was constant and distractor color changed or remained constant as well. Their finding, that repetition of distractor colors increased search performance, was the first indicator that distractor suppression may also benefit from priming of features. Later studies systematically varied target and distractor features and showed that the relative contribution of target and distractor feature repetition to intertrial priming both seem to be additive (Lamy et al., 2008) while swapping features may induce additional costs (Kristjánsson \& Driver, 2008). In line with this, a neurophysiological study that systematically varied target and distractor feature repetition, showed that the $\mathrm{N} 2 \mathrm{pc}$, indicative of attention deployment toward the target, was equally pronounced for target and distractor repetition (Eimer et al., 2010). This suggests that target and distractor feature repetition may contribute equally to intertrial priming due to comparable modulation of attention deployment toward the target in case one of both remains constant between succeeding trials.

It is still unclear how distractor repetition facilitates suppression of the distractor. A clinical study showed that the search performance of patients with visual hemispatial neglect benefited from repetition of distractor colors (Saevarsson, Jóelsdóttir, Hjaltason, \& Kristjánsson, 2008). Another clinical study showed that when frontal eye fields are affected, intertrial priming of distractor features was absent (Finke et al., 2009). These results indicate that distractor suppression is facilitated at early stages of visual processing when distractor features are repeatedly presented.

Additionally, there is evidence that intertrial priming is not purely automatic (Hillstrom, 2000; Leonard \& Egeth, 2008; Müller, Krummenacher, \& Heller, 2004). For example, in addition to varying whether a feature was repeated, Hillstrom (2000) varied whether a feature change occurred randomly or according to an alternating pattern that allowed observers to predict the upcoming feature. She found that predictability can potentiate intertrial priming, suggesting that intertrial priming can be modulated by volitional control. In line with this, while it was shown that intertrial priming at the feature level (e.g., red vs. blue) operates in an automatic manner (Geyer et al., 2006; see also Maljkovic \& Nakayama, 1994, Experiment. 4), it was also shown that intertrial priming at the dimension level (e.g., color vs. shape) is more penetrable by volitional control (Fecteau, 2007; Müller et al., 2004). For example, Fecteau found that repetition of the target dimension (color vs. shape) only improves performance if the target dimension is relevant to the observer's current goals. The present study used an approach similar to Hillstrom (2000); however, we systematically varied predictability and repetition of distractor features, rather than target features, as was used by Hillstrom.

Concerning the mechanism underlying intertrial priming of target features, it has been suggested that perceptual processing of previously attended objects or features is facilitated, that sensitivity for certain features is increased, and that attention deployment is faster toward stimuli that share features with previously relevant items (for a review, see Kristjánsson \& Campana, 2010). For intertrial priming of distractor features, the mechanism driving a faster search for targets while irrelevant features are repeated is less clear. According to the dimensional-weighting account (Found \& Müller, 1996; Müller et al., 2004), high weights for task-relevant features and lower weights for task-irrelevant features can carry over to subsequent trials in an automatic manner. Becker et al. (2009) suggested that dimensional weighting can account for intertrial priming; targets receive higher activation in a given trial if the same target was shown in the previous trial. Analogously, it seems plausible that salient distractors are less likely to capture attention if their features still "suffer" from low weights spilling over from the recent selection history. If that was the case, signals that enhance volitional control in a trial should more likely overrule attentional weights from previous trial activation, which, in turn, should result in reduced or no intertrial priming. Context heterogeneity may be one way of modulating the ratio of volitional control and salience-based processing. More recently, we showed that prioritization of targets and suppression of salient distractors (indicative of volitional control; see Sawaki \& Luck, 2013) was achieved faster in homogeneous compared to heterogeneous contexts, while attention capture by a salient distractor (indicative of salience-based processing; see Theeuwes, 2004) was only observed in heterogeneous contexts (FeldmannWüstefeld \& Schubö, 2013). Distractor-related attentional capture in heterogeneous contexts was even more prominent if distractors were rewarded (Feldmann-Wüstefeld, Brandhofer, \& Schubö, 2016). These results showed that when the visual properties of the visual field are favorable (as in homogeneous contexts), targets can be easily and rapidly found, thus keeping bottom-up-driven attention deployment to a minimum. When the visual properties of the visual field are less favorable (as in heterogeneous contexts), the target is harder to find, and any distractor, especially a salient one, is more likely to be accidentally selected during inefficient search because of attentional misguidance (Todd \& Kramer, 1994), undermining focused top-down attention deployment. In the present study, we will use a similar context homogeneity manipulation to investigate whether increased efficiency of attention deployment after distractor feature 
repetition can be modulated by a visual context that allows a varying amount of volitional control.

\section{Rationale of the present study}

The present study investigated whether the predictability and repetition of a feature defining a task-irrelevant but salient distractor feature facilitates visual processing. We expected that repetition of distractor features should speed up visual search through intertrial priming. We also expected to find more efficient visual search in trials in which observers expected a certain distractor feature to appear, possibly even more so if the distractor feature was repeated. Importantly, we were not only interested in improved suppression of the distractor itself but also in improved processing of the taskrelevant target that was entirely unrelated to the distractors. To disentangle these effects, a systematic lateralization of target and distractor was used (Hickey, Di Lollo, \& McDonald, 2009; Hickey, Olivers, Meeter, \& Theeuwes, 2011; Woodman \& Luck, 2003; see also Munneke, Fait, \& Mazza, 2013; Sawaki \& Luck, 2010). Presenting the target laterally with the distractor on the vertical midline (i.e., nonlaterally) allows isolating the $\mathrm{N}_{\mathrm{T}}$ (the negative part of the $\mathrm{N} 2 \mathrm{pc}$ contralateral to targets) component reflecting target-related activity in the event-related potential (ERP) of the EEG (Hickey et al., 2009; Hilimire, Hickey, \& Corballis, 2012). Conversely presenting the distractor laterally with the target on the vertical midline allows isolating the $\mathrm{N}_{\mathrm{D}}$ (the negative part of the $\mathrm{N} 2 \mathrm{pc}$ contralateral to distractors) component reflecting attentional capture by the distractor (Kiss, Grubert, Petersen, \& Eimer, 2012; Sawaki \& Luck, 2010) and the $P_{D}$ (the positive part of the N2pc ipsilateral to distractors) component reflecting active suppression of the distractor (Burra \& Kerzel, 2013; Feldmann-Wüstefeld \& Schubö, 2013; Hickey et al., 2009; Sawaki, Geng, \& Luck, 2012). Moreover, target and distractor will be embedded in either a homogenous or heterogeneous context. Because heterogeneous contexts were shown to be more prone to attentional capture than were homogeneous contexts, we expected to find both expectancy and repetition of distractor features to facilitate visual search, in particular, in heterogeneous contexts. Both expectancy and repetition of distractor features are likely to improve efficiency in deploying attention to the target in an otherwise bottom-updominated visual setting.

\section{Method}

\section{Participants}

Seventeen volunteers (3 male), ages $18-27$ years $(M=22.0$, $S D=2.4)$, all right-handed, naive to the paradigm and objective of the experiment, participated for payment or course credit in Experiment 1. Twenty-eight new volunteers, naive to the paradigm and objective of the experiment, participated for payment or course credit in Experiment 2. Six participants had to be excluded from analysis in Experiment 2 because of excessive eye-related artifacts (see below for criteria). Of the remaining 22 participants (10 male), ages 19-30 years $(M=22.5, S D=2.9)$, all but three were righthanded. Participants in both experiments had normal or corrected-to-normal vision and no color blindness (all tested with Oculus Binoptometer 3). Both experiments were conducted with the written understanding and consent of each participant.

\section{Stimuli and apparatus}

Participants were seated in a comfortable chair in a dimly lit, electrically shielded, and sound attenuated chamber, with an ergonomic gamepad (Microsoft Sidewinder Plug \& Play USB) in their hands. Participants had to use their left and right index finger to press two buttons on the back of the game pad. Stimulus presentation and response collection were controlled by a Windows PC using E-Prime 2 routines. All stimuli were presented on a LCD-TN screen (Samsung Syncmaster 2233) that was located $100 \mathrm{~cm}$ from the eyes of participants. Search displays with 458 line elements arranged in a matrix of $27 \times$ 17 stimuli were shown on a gray (RGB: 60, 60, 60) background. In the central position, a fixation dot was shown instead of a line element. Single lines had a length of $0.7^{\circ}$ of visual angle, and the matrix's dimension were $24.2^{\circ} \times 16.2^{\circ}$. The target was a gray $(134,134,134)$ oblique line element tilted $45^{\circ}$ either to the left or right (equiprobably). The color singleton distractor was a red $(232,34,34)$ or blue $(82,24$, 255 ) horizontal or vertical line (colors were matched for luminance with a Minolta LS-110 luminance meter). The remaining 456 line elements were gray $(134,134,134)$ horizontal or vertical lines. In the homogeneous condition, all of the remaining 456 line elements (and the color singleton) were either horizontal or vertical (changing randomly from trial to trial. cf. Fig. 1a). In the heterogeneous condition, 228 of the remaining lines were horizontal and 228 were vertical, randomly assigned to the matrix positions (cf. Fig. 1b). In each trial, the target and the color singleton appeared at two out of six equiangular positions on an imaginary circle (cf. Fig. 1). Two of the positions were $5.8^{\circ}$ above or below fixation on the vertical midline. The other four positions were $4.9^{\circ}$ left or right of the vertical midline and $2.9^{\circ}$ above or below the horizontal midline. In one third of the trials, the target was presented in one of the vertical midline positions, and the singleton was presented in a neighboring lateral position. In another third of the trials, this was reversed and in the remaining third of the trials, both were presented in a lateral position, namely in opposite hemifields. 
a Homogeneous context

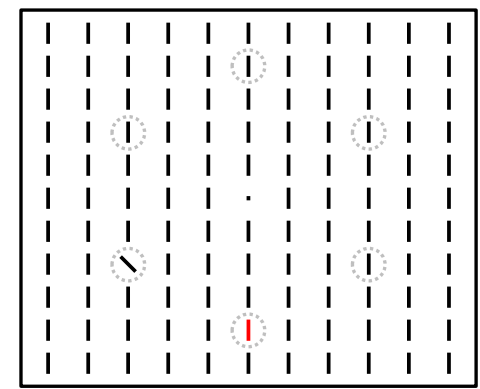

C Block 1

Block 2

Heterogeneous

Random order

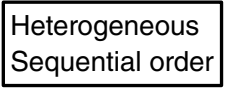

RRBRBBBRB...

BBBRRRBBB.. b Heterogeneous context

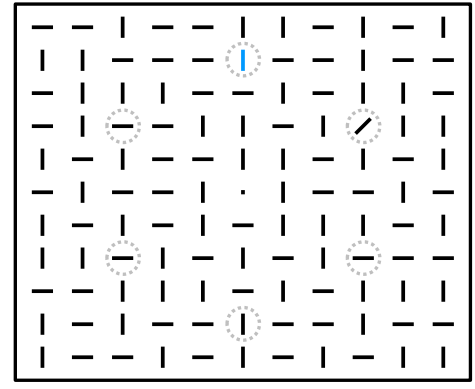

Block 3

Homogeneous

Block 4

Random order

Homogeneous

Sequential order

BRRRBRBBR... BBBRRRBBB...
Fig. 1 a and b show exemplary contexts used as search displays. Participants had to search for an oblique line and report its orientation (leftward vs. rightward). The target was embedded in a homogeneous context (a), in which distractors were all horizontal or all vertical lines, or in heterogeneous context (b), in which horizontal and vertical lines were randomly distributed. There was always an additional color singleton (red vs. blue) embedded in the context that was irrelevant to observers. To disentangle target and (color-) distractor processing in the lateralized EEG, the target was presented laterally with a nonlateral distractor (shown here) or the distractor was presented laterally with a

\section{Procedure}

A trial started with the presentation of a gray central fixation dot that remained on the screen throughout the entire trial. After $500 \mathrm{~ms}$, the search display was presented for $200 \mathrm{~ms}$ and then replaced with a blank display. Participants were asked to press one of the response buttons (/ or $\backslash)$ in order to indicate the orientation of the target in the search display (i.e., whether the target was pointing right upwards or right downwards). Button assignment was balanced across participants. Response speed was emphasized, but there was no time limit for the response. After their response, the fixation dot disappeared for 1,000 ms until a new fixation dot announced the start of a new trial. Participants were told that both the context (the 456 horizontal and vertical lines) and the color singletons were irrelevant to the task and could be ignored.

There were 1,152 trials (1,440 in Experiment 2) in total, divided into 16 blocks (20 in Experiment 2) of 72 trials. Target and singleton location and target identity were randomly selected in every trial, whereas context type (homogeneous and heterogeneous) was fixed for two blocks and predictability (predictable vs. unpredictable distractor color) was alternating from block to block. In predictable blocks, distractor color (blue or red) systematically alternated in chunks of three colors in a row (e.g., red, red, red, blue, blue, blue; order counterbalanced across participants). In unpredictable blocks, distractor identity was randomly chosen in each trial. An exemplary block pattern would be: Het-Sequ, Het-Rand, HomSequ, Hom-Rand. One additional block in the beginning of the nonlateral target (not shown here). For illustration purposes, the number of lines was reduced in this figure; the actual number of lines was 458 . Lines were light gray on a dark gray background. c shows exemplary pattern of distractor predictability and context homogeneity. In unpredictive blocks, distractor color (R-Red, B-Blue) varied randomly from trial to trial. In predictive blocks, distractor color varied systematically in chunks of three presentations in a row. Context homogeneity varied every two blocks, and predictability alternated between each block. (Color figure online.)

experiment served as training. After every block, performance feedback (response times and accuracy) was given to participants. Participants had to take a break of at least $10 \mathrm{~s}$ in the middle of each block and after each block (i.e., each 36 trials).

\section{EEG recording}

In addition to RTs and accuracy, in Experiment 2, EEG was recorded with $\mathrm{Ag}-\mathrm{AgCl}$ active electrodes (BrainProducts actiCap) from 64 scalp sites (according to the International 10/20 System). Horizontal and vertical EOGs were recorded bipolarly from the outer canthi of the eyes and from above and below the observer's left eye, respectively. All electrodes were referenced to $\mathrm{Cz}$ and re-referenced off-line to the average of all electrodes. Electrode impedances were kept below $5 \mathrm{k} \Omega$. Sampling rate was $1000 \mathrm{~Hz}$ with a high cutoff filter of $250 \mathrm{~Hz}$ and a low cutoff filter of $0.016 \mathrm{~Hz}$ (half power cutoff, $24 \mathrm{~dB}$ roll-off).

\section{Data analysis}

Behavioral data Mean response times (RT) and accuracy were calculated separately for each participant for Context type, Order, and Predictability. Trials with exceedingly long RT ( $>1,000 \mathrm{~ms})$ were removed from RT and accuracy analyses. Trials with false responses were removed from the RT analysis. There were relatively few trials in which distractor identity was repeated three times in a row in random order trials because of the randomized repetition/nonrepetition, which is why second and third 
presentation were binned for random order trials. To match the factor levels of Order between random order trials and sequential order trials, the factor Order was analyzed on two levels for the initial analysis (first vs. second/third presentation).

EEG data (Experiment 2 only) EEG was averaged off-line over an 800-ms epoch, including a 200-ms prestimulus baseline with epochs time locked to search display onset. Trials with incorrect responses or exceedingly long RTs $(>1,000 \mathrm{~ms})$ were excluded from analysis. To identify eye-related artifacts, channel FP1 was subtracted from a channel below the left eye (=vEOG), and channel F10 was subtracted from channel F9 $(=$ hEOG) and then low-pass filtered (Butterworth, $20 \mathrm{~Hz}$ half amplitude cutoff, $24 \mathrm{~dB}$ roll-off). Trials in which vEOG revealed blinks (indicated by an absolute voltage exceeding $\pm 80 \mu \mathrm{V}$ ) or hEOG revealed eye movements (indicated by an absolute voltage exceeding $\pm 30 \mu \mathrm{V}$ ) were removed from analyses. For six participants, more than $25 \%$ of the trials had to be removed from analyses, and these participants were excluded from data analysis. Of the remaining 22 subjects, $93.4 \%(S D=6.4 \%)$ of the trials showed no signs of eye-related artifacts. Additionally, segments were excluded from further analysis on an individual-channel basis when the absolute voltage exceeded $\pm 80 \mu \mathrm{V}$.

ERP analysis epochs for $\mathrm{N}_{T}, \mathrm{~N}_{\mathrm{D}}$, and $\mathrm{P}_{\mathrm{D}}$ were determined separately for predictable and unpredictable blocks. This was done by collapsing the mean lateralized ERP (activity contralateral to lateralized target / distractor minus activity ipsilateral to lateralized target / distractor) of the electrode pairs PO7/PO8 and PO3/PO4 across Context types and Order ("condition blind"), resulting in two waveforms for trials with lateral targets and two waveforms for trials with lateral color singletons. Epochs were determined as the time $\pm 20 \mathrm{~ms}$ around the peak of the lateralized components (rounded to 5-ms steps). The $\mathrm{N}_{\mathrm{T}}$ peaked around $195 \mathrm{~ms}$ (epoch: 175-215 ms) in predictable and around $200 \mathrm{~ms}$ (epoch: 180-220 ms) in unpredictable trials. The $\mathrm{N}_{\mathrm{D}}$ peaked around $200 \mathrm{~ms}$ (epoch: 180-220 ms) for predictable and unpredictable trials. The $\mathrm{P}_{\mathrm{D}}$ peaked around $260 \mathrm{~ms}$ (epoch: 240-280 ms) for predictable and unpredictable trials. Subsequently, mean lateralized ERP activity (PO7/PO8/PO3/ PO4) was calculated for the epochs of the respective laterality condition, separately Context type and Order (first, second, and third for predictable blocks; first and second or more for unpredictable blocks; see above).

\section{Results}

\section{Behavioral data, Experiment 1 (see Fig. 2a)}

Response times were analyzed in a three-way ANOVA with the following factors: Context type (heterogeneous vs. homogeneous), Order (first vs. second/third presentation) and Predictability (predictable vs. unpredictable). Response times were faster in homogeneous $(M=472 \mathrm{~ms})$ than in heterogeneous contexts $(M=507 \mathrm{~ms}), F(1,16)=174.2, p<.001$, $\eta^{2}=.916$. Response times were also faster for the second or third presentation of a distractor $(M=487 \mathrm{~ms})$ than for the first presentation of a distractor $(M=492 \mathrm{~ms}), F(1,16)=11.6$, $p=.004, \eta^{2}=.420$, which constitutes an intertrial priming effect. Intertrial priming was more pronounced in heterogeneous contexts $(\Delta M=9 \mathrm{~ms})$ than in homogeneous contexts $(\Delta M-5 \mathrm{~ms}), F(1,21)=4.9, p=.037, \eta^{2}=.191$. Follow-up $t$ tests across order (first, second, third) showed that response times were shorter for predictable than for unpredictable blocks for heterogeneous contexts $\left(M_{\text {pred }}=503 \mathrm{~ms}\right.$ vs. $\left.M_{\text {unpred }}=512 \mathrm{~ms}\right), t(16)=4.0, p=.001$, but not for homogeneous contexts $\left(M_{\text {pred }}=474 \mathrm{~ms}\right.$ vs. $\left.M_{\text {unpred }}=469 \mathrm{~ms}\right)$, $t(16)=1.9, p=.078$.

\section{Behavioral data, Experiment 2 (see Fig. 2b)}

The same ANOVA as in Experiment 1 was run. Response times were faster in homogeneous $(M=500 \mathrm{~ms})$ than in heterogeneous contexts $(M=536 \mathrm{~ms}), F(1,21)=94.8, p<.001$, $\eta^{2}=.819$. Response times were also faster for the second or third presentation of a distractor $(M=516 \mathrm{~ms})$ than for the first presentation of a distractor $(M=520 \mathrm{~ms}), F(1,21)=6.1, p=$ $.022, \eta^{2}=.226$, which constitutes an intertrial priming effect. There was a trend for faster response times in predictable $(M=$ $516 \mathrm{~ms})$ compared to unpredictable blocks $(M=520 \mathrm{~ms}), F(1$, $21)=3.9, p=.061, \eta^{2}=.157$. The intertrial priming was more pronounced in heterogeneous contexts $(\Delta M=5 \mathrm{~ms})$ than in homogeneous contexts $(\Delta M=1 \mathrm{~ms}), F(1,21)=4.9, p=.037$, $\eta^{2}=.191$. Furthermore, the advantage for predictable blocks was pronounced in heterogeneous contexts $(\Delta M=9 \mathrm{~ms})$ and absent in homogeneous contexts $(\Delta M=-2 \mathrm{~ms})$, interaction of Predictability and Context type, $F(1,21)=18.1, p<.001, \eta^{2}=$ .462 . No other effects were significant (all $p \mathrm{~s}>.155$ ).

Follow-up $t$ tests across predictable/unpredictable blocks showed that response times decreased from first to second/ third distractor presentation for heterogeneous contexts $\left(M_{1 \mathrm{st}}\right.$ $=539 \mathrm{~ms}$ vs. $\left.M_{2 \mathrm{nd} / 3 \mathrm{rd}}=534 \mathrm{~ms}\right), t(21)=4.9, p<.001$, but not for homogeneous contexts $\left(M_{1 \mathrm{st}}=500 \mathrm{~ms}\right.$ vs. $M_{2 \mathrm{nd} / 3 \mathrm{rd}}=$ $499 \mathrm{~ms}), t(21)=0.8, p=.441$.

Follow-up $t$ tests across order (first, second, third) showed that response times were shorter for predictable than for unpredictable blocks for heterogeneous contexts $\left(M_{\text {pred }}=532 \mathrm{~ms}\right.$ vs. $\left.M_{\text {unpred }}=541 \mathrm{~ms}\right), t(21)=4.8, p<.001$, but not for homogeneous contexts $\left(M_{\text {pred }}=501 \mathrm{~ms}\right.$ vs. $\left.M_{\text {unpred }}=499 \mathrm{~ms}\right)$, $t(21)=0.7, p=.496$. Accuracy was not modulated by context, predictability or order (all $p \mathrm{~s}>.302)$.

\section{EEG data, Experiment 2}

Three-way ANOVAs were calculated separately for $\mathrm{N}_{\mathrm{D}}, \mathrm{P}_{\mathrm{D}}$ and $\mathrm{N}_{\mathrm{T}}$. Each individual ANOVA included the following 


\section{Experiment 1}

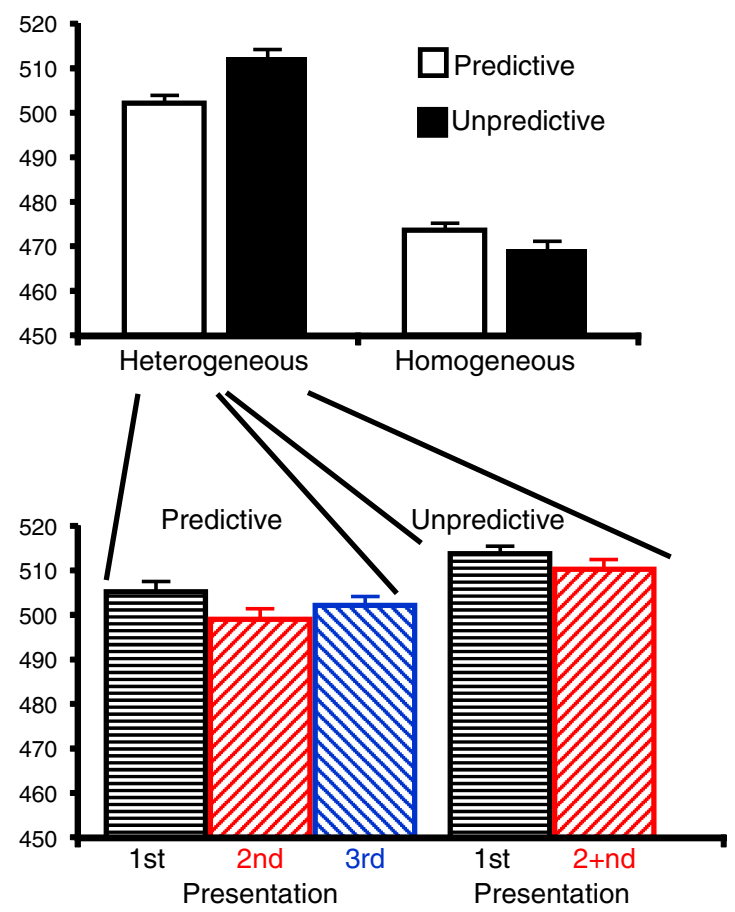

Fig. 2 a shows mean RTs for targets embedded in heterogeneous and homogeneous contexts, separately for predictable blocks, in which color distractors systematically varied in chunks of three repetitions (e.g., red, red, red, blue, blue, blue), and for unpredictive blocks, in which color distractors varied randomly from trial to trial (e.g., red, blue, blue, red, blue, red, red). b shows mean RTs in heterogeneous contexts, separately

factors: Context type (heterogeneous vs. homogeneous), Predictability (predictable vs. unpredictable), and Order (first presentation vs. second/third presentation). The reliability of lateralized components was tested by conducting four (Context Type $\times$ Predictability, across Order) one-tailed $t$ tests against zero, separately for $\mathrm{N}_{\mathrm{D}}, \mathrm{P}_{\mathrm{D}}$, and $\mathrm{N}_{\mathrm{T}}$. All three components showed a mean amplitude significantly different from zero in all four conditions (all $t$ tests reached $p$ values $\leq .004$ ).

$\mathrm{N}_{\mathrm{D}}$ data (distractor lateral, target nonliteral; see Figs. 3 \& 4) An interaction of Context type and Order showed that the $\mathrm{N}_{\mathrm{D}}$ was modulated differently by Order in homogeneous and heterogeneous contexts, $F(1,21)=10.0, p=.005, \eta^{2}=.322$. An interaction of Predictability and Order showed that the $\mathrm{N}_{\mathrm{D}}$ was modulated differently by Order in predictable and unpredictable trials, $F(1,21)=8.7, p=.008, \eta^{2}=.293$. Furthermore, Context, Order, and Predictability showed a three-way interaction, $F(1,21)=7.5, p=.012, \eta^{2}=.263$. To further explore the data, follow-up ANOVAs with the factors Context and Order were computed separately for predictable and unpredictable trials.

Predictable distractors Because there were sufficient data points for second and third presentation separately, the

\section{Experiment 2}

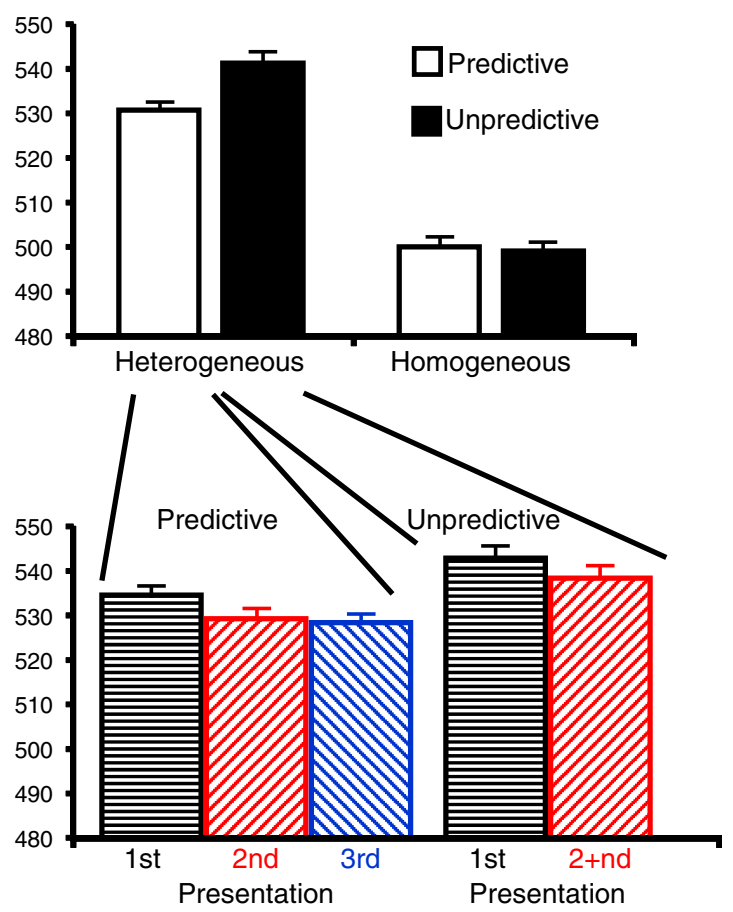

for the first, second, and third presentation of a distractor color in a row (for unpredictive blocks, second and more presentations were pooled because too few data were available for individual analysis). Error bars denote the standard error of the mean corrected for between-subjects variance (Cousineau, 2005). (Color figure online.)

factor Order was analyzed on three levels (first, second, third presentation). There was no main effect of Context $(p=.110)$ or Order $(p=.720)$. An interaction of Context and Order showed that the $\mathrm{N}_{\mathrm{D}}$ was modulated differently by Order in heterogeneous (see Fig. 3, left column) and homogeneous (see Fig. 3, right column) contexts, $F(2$, 42) $=9.3, p=.002, \eta^{2}=.307$. Follow-up ANOVAs for each context separately showed that the $\mathrm{N}_{\mathrm{D}}$ was modulated by Order in heterogeneous contexts $\left(M_{1 \mathrm{st}}=\right.$ $\left.-1.04 \mu \mathrm{V}, M_{2 \text { nd }}=-0.65 \mu \mathrm{V}, M_{3 \mathrm{rd}}=-0.35 \mu \mathrm{V}\right), F(2$, $42)=4.8, p=.013, \eta^{2}=.187$, and homogeneous contexts $\left(M_{1 \mathrm{st}}=-0.17 \mu \mathrm{V}, M_{2 \mathrm{nd}}=-0.57 \mu \mathrm{V}, M_{3 \mathrm{rd}}=\right.$ $-0.66 \mu \mathrm{V}), F(2,42)=4.7, p=.015, \eta^{2}=.182$. Planned contrast revealed that in heterogeneous contexts, $\mathrm{N}_{\mathrm{D}}$ was larger for first distractor presentation than for second, $t(21)=1.9, p=.038$, or third, $t(21)=2.6, p=$ .009 , presentation, but of similar size for second and third presentation, $t(21)=1.7, p=.055$, which constitutes an intertrial priming effect (see Fig. 3, left column). In homogeneous contexts, $\mathrm{N}_{\mathrm{D}}$ was smaller for first distractor presentation than for second, $t(21)=2.2, p=.020$, or third, $t(21)=2.6, p=.009$, presentation, but of similar size for second and third presentation, $t(21)=0.7, p=$ .246 (see Fig. 3, right column). 
Heterogeneous

First

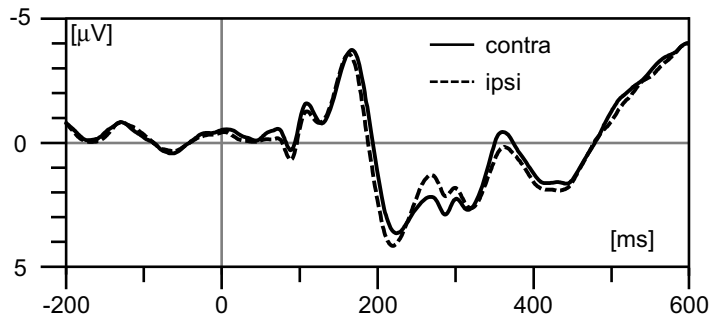

Second

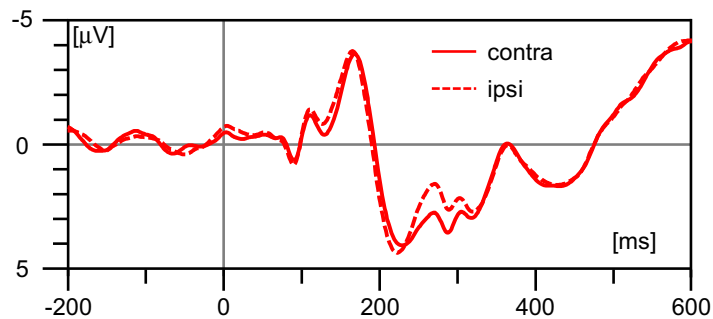

Third

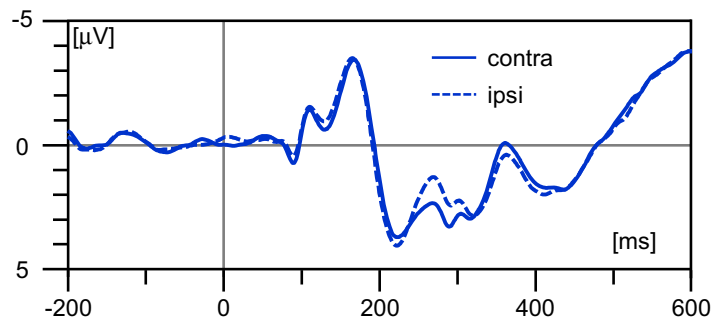

Difference

Waves

(contra-ipsi)

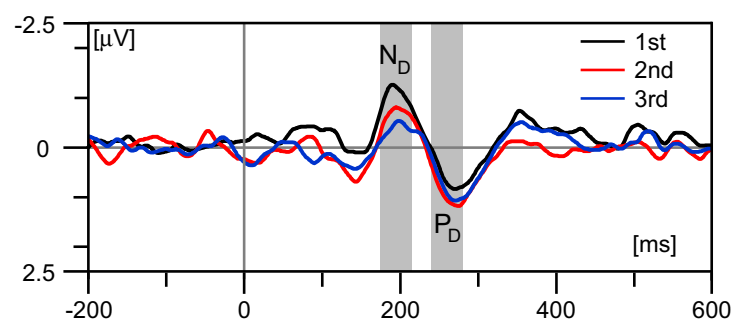

Homogenoeus
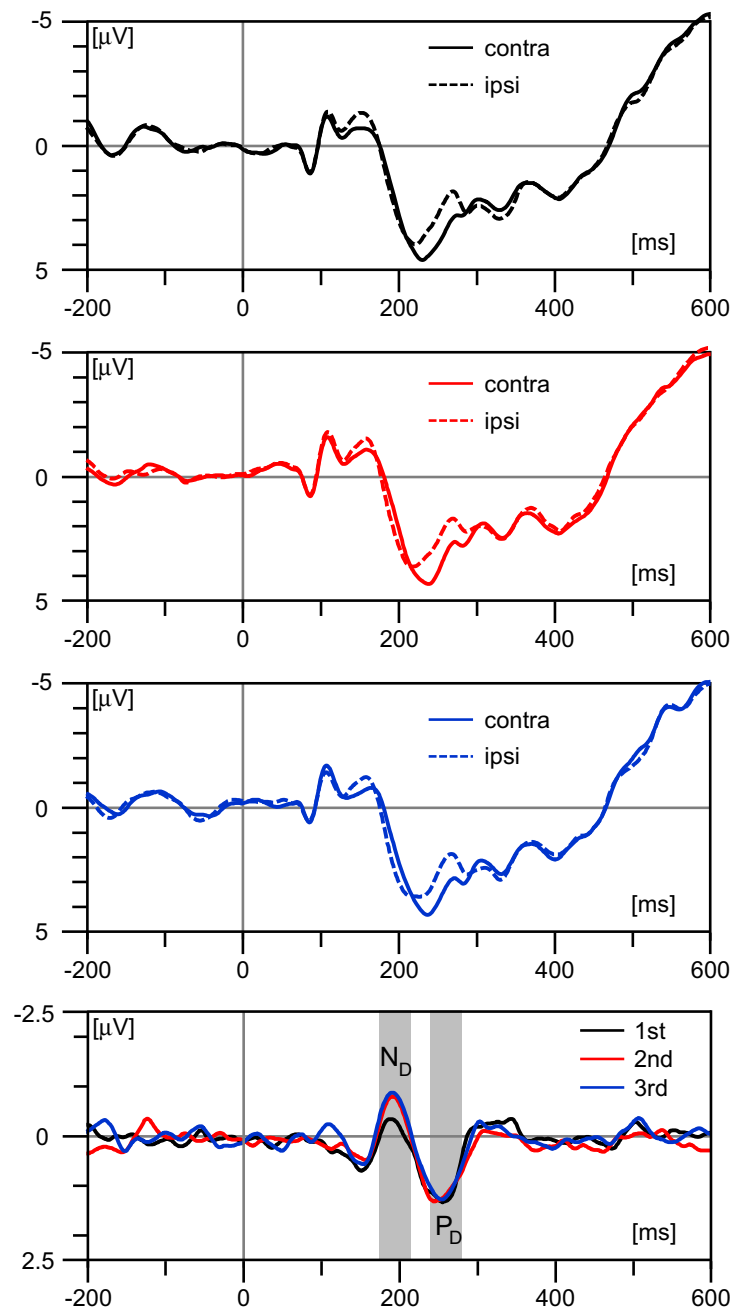

Fig. 3 Predictable order, distractors lateral. Grand average ERPs recorded at posterior-occipital electrode sites (pool of PO3/4 and PO7/ 8 ), elicited by search displays for trials with heterogeneous contexts (left column) and trials with homogeneous contexts (right column) in trials with lateral distractors in predictable blocks. The first row shows trials in which a distractor is presented the first time in a series. The second and

Unpredictable distractors Because there were not sufficient data points for the third presentation, the factor Order was analyzed on two levels (first, second/third presentation). There were no main effects or interaction (all $p$ s $>$.279; see Fig. 4).

$\mathbf{P}_{\text {D }}$ data (distractor lateral, target nonliteral; see Figs. 3 \& 4) The same ANOVA as for $N_{D}$ was calculated and revealed no main effects or interactions (all $p s>.072$ ).

$\mathbf{N}_{\text {T }}$ data (target lateral, distractor nonliteral; see Figs. 5 \& 6) The same ANOVA as for $N_{D}$ and $P_{D}$ was calculated and revealed that the $\mathrm{N}_{\mathrm{T}}$ was smaller in heterogeneous $(-1.10 \mu \mathrm{V})$ than in homogeneous contexts $(-1.96 \mu \mathrm{V}), F(1,21)=22.2, p$ $<.001, \eta^{2}=.514$. There were no other effects (all $p \mathrm{~s}>.167$ ). third rows show data for second and third distractor color presentation, respectively. The bottom row shows the same data as in rows $1-3$ but as difference waves (contra minus ipsi in relation to the distractor). Epochs for statistical analyses are shaded gray. For illustration purposes, EEG waveforms were low-pass filtered at $30 \mathrm{~Hz}$ (half power cutoff, $24 \mathrm{~dB}$ ) using digital filtering. (Color figure online.)

\section{Discussion}

In the present study, we investigated whether predictability and repetition of a task-irrelevant but salient distractor facilitates visual processing. We found that the repetition of features across trials can facilitate visual processing, an effect previously described as intertrial priming (Maljkovic \& Nakayama, 1994; see also Becker et al., 2009; Eimer et al., 2010; Found \& Müller, 1996; Kristjánsson et al., 2007). Specifically, we repeated the color of irrelevant distractor items that were potentially interfering with target processing. The key finding of the present study was that faster visual search for a target through repetition of a distractor was only observed under two prerequisites: (1) The distractor repetition was systematic rather than random and (2) the distractor was embedded in a 
Heterogeneous

First

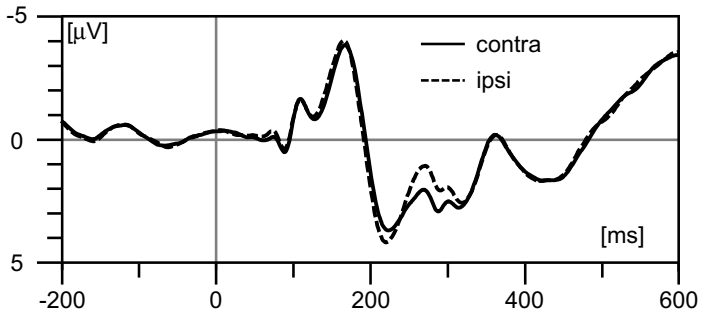

Second +

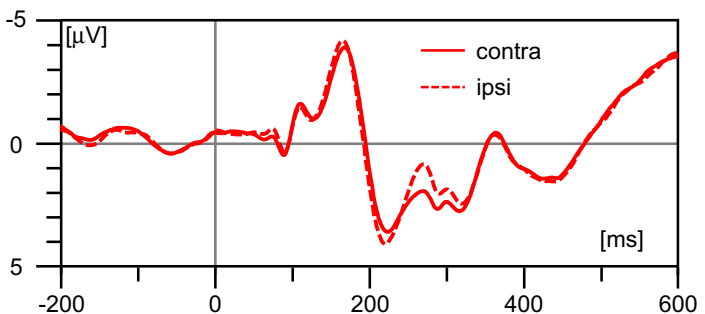

Difference

Waves (contra-ipsi)

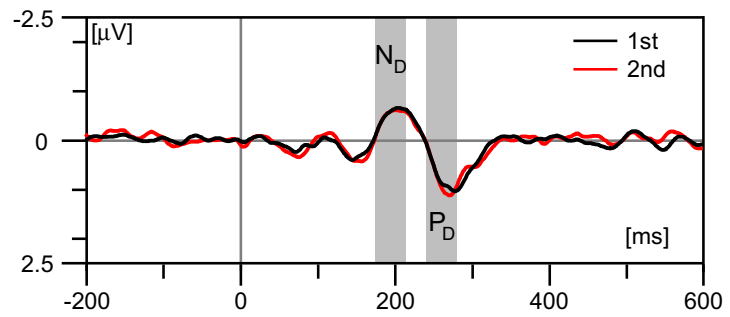

Homogenoeus
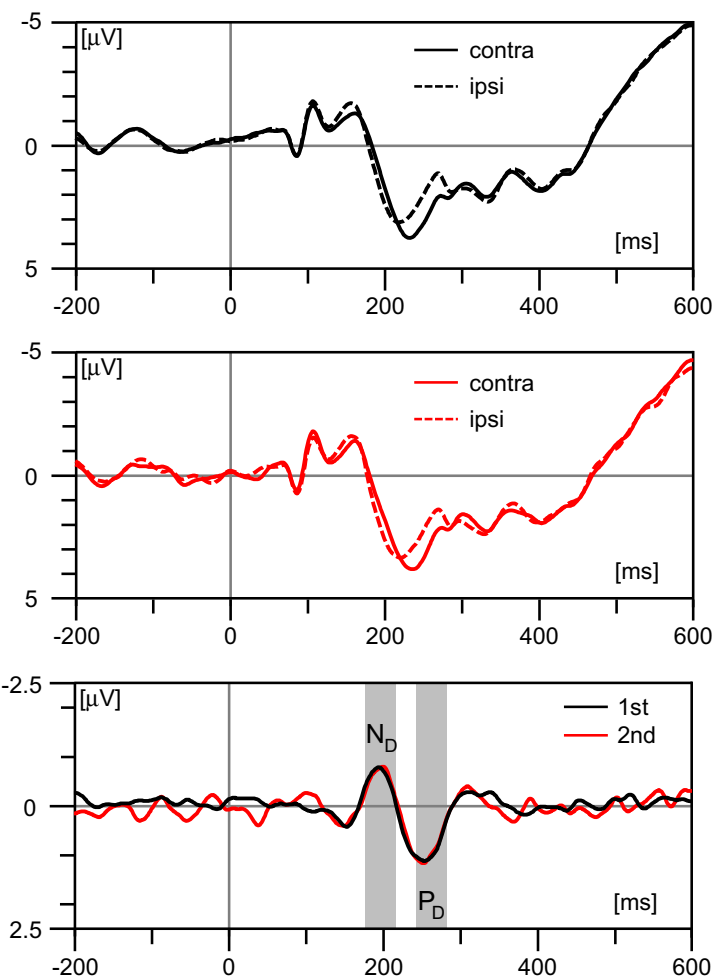

Fig. 4 Unpredictable order, distractors lateral. Grand average ERPs recorded at posterior-occipital electrode sites (pool of PO3/4 and PO7/ 8 ), elicited by search displays for trials with heterogeneous contexts (left column) and trials with homogeneous contexts (right column) in trials with lateral distractors in unpredictable blocks. The first row shows trials in which a distractor is presented the first time in a series. The second row shows data for second and more distractor color presentation. The bottom row shows the same data as in rows 1-2 but as difference waves (contra minus ipsi in relation to the distractor). Epochs for statistical analyses are shaded gray. For illustration purposes, EEG waveforms were low-pass filtered at $30 \mathrm{~Hz}$ (half power cutoff, $24 \mathrm{~dB}$ ) using digital filtering

that intertrial priming for distractors only occurs if the distractor feature varies systematically rather than randomly.

We measured event-related potential (ERPs) of the EEG in order to investigate the underlying mechanism for the differential efficacy of intertrial priming. By systematically varying laterality of target and distractor, we were able to disentangle target processing from distractor processing (see Hickey et al., 2009). ERP results were in line with behavioral data and reveal why intertrial priming was only observed under certain circumstances; the $\mathrm{N}_{\mathrm{T}}$ was larger in homogeneous than in heterogeneous contexts, a replication of earlier findings (e.g., Feldmann-Wüstefeld et al., 2016; Feldmann-Wüstefeld \& Schubö, 2013), showing that prioritization of relevant information is easier in homogeneous contexts. Most interestingly, the $\mathrm{N}_{\mathrm{T}}$ was modulated neither by expectation nor by repetition; participants could neither prioritize the target more easily when a distractor color was repeated nor when a distractor color was expected. This suggests that target processing itself was not directly affected by statistical or physical properties of the distractor.

The distractor-elicited $\mathrm{N}_{\mathrm{D}}$ component that is indicative of attentional capture, however, was modulated by expectation 
Heterogeneous

First

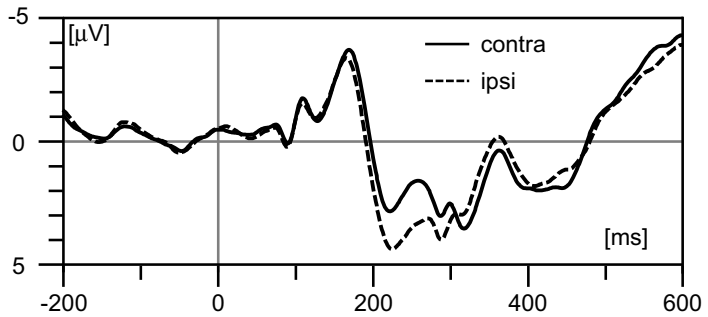

Second

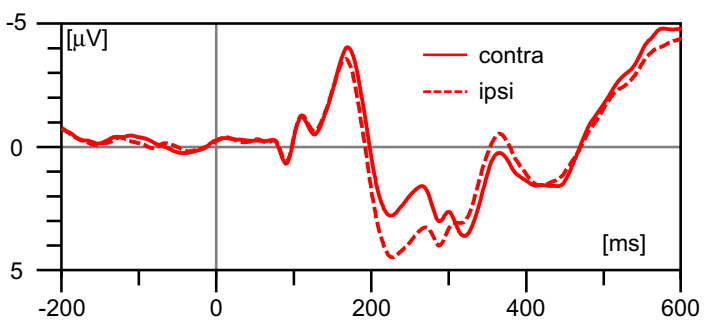

Third

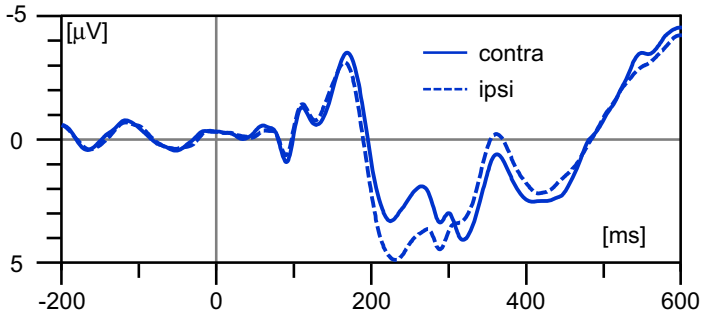

\section{Difference \\ Waves (contra-ipsi)}

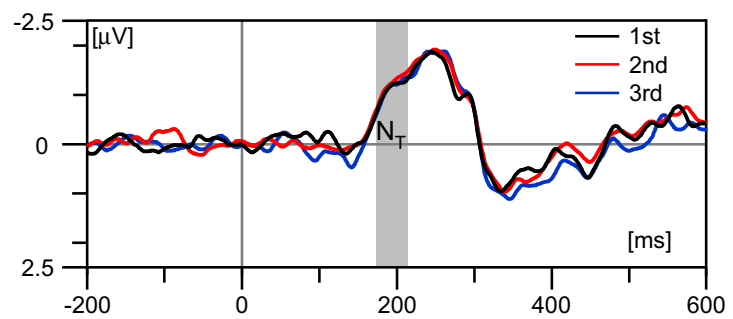

Homogenoeus
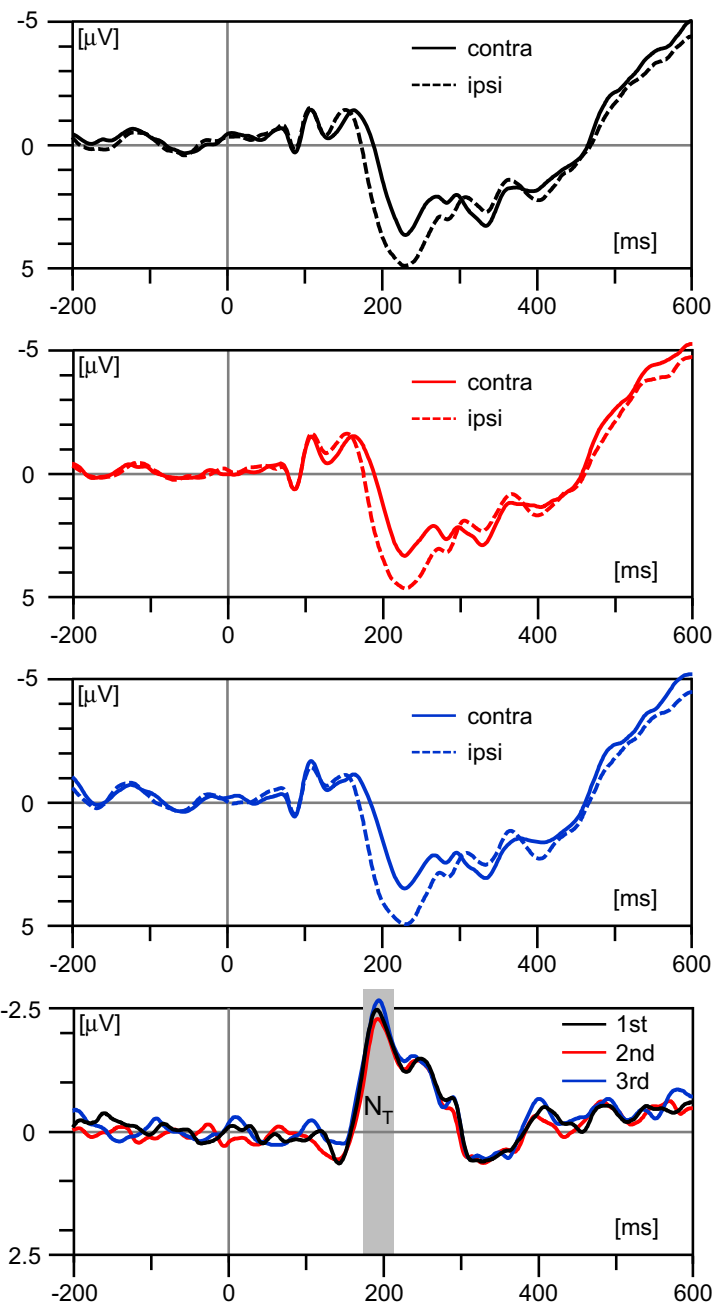

Fig. 5 Predictable order, targets lateral. Grand average ERPs recorded at posterior-occipital electrode sites (pool of PO3/4 and PO7/8), elicited by search displays for trials with heterogeneous contexts (left column) and trials with homogeneous contexts (right column) in trials with lateral targets in predictable blocks. The first row shows trials in which a distractor is presented the first time in a series. The second and third

and repetition. When feature color varied unpredictably from trial to trial, the $\mathrm{N}_{\mathrm{D}}$ did not vary as a function of repetition, either in homogeneous or in heterogeneous contexts (but note that RTs decreased for repeated distractor presentation in both predictable and unpredictable blocks). When distractor color varied systematically, so that observers could predict when a particular color could be ignored, the $\mathrm{N}_{\mathrm{D}}$ decreased with distractor repetition, but only in heterogeneous contexts. The $\mathrm{N}_{\mathrm{D}}$ was largest for the first presentation of a distractor, followed by the second presentation, and was smallest for the third presentation. This suggests that when a particular distractor color was used the first time in a sequence of repetitions, this distractor captured attention more likely than when the same distractor was repeated once or twice. In line with this, it was found that colors that defined a target in a previous trial lead to rows show data for second and third distractor color presentation, respectively. The bottom row shows the same data as in rows 1-3 but as difference waves (contra minus ipsi in relation to the target). Epochs for statistical analyses are shaded gray. For illustration purposes, EEG waveforms were low-pass filtered at $30 \mathrm{~Hz}$ (half power cutoff, $24 \mathrm{~dB}$ ) using digital filtering

a pronounced $\mathrm{N}_{\mathrm{D}}$ elicited by distractors bearing the previously target-defining feature in the current trial (target and distractor features swapped) but not when the distractor feature remained the same (Hickey et al. 2011). These results suggest that distractor feature repetition causes a devaluation of distractor features in a subsequent trial.

In the present study, in homogeneous contexts, $\mathrm{N}_{\mathrm{D}}$ was smallest for first distractor presentation and increased for repetitions. It is also possible that the smaller negativity in the $\mathrm{N}_{\mathrm{D}}$ epoch for first compared to second third presentation is due to an early $P_{D}$ in a subset of trials, which makes the lateral deflection relatively positive in average. It is unclear why this apparent decrease of attentional capture for first distractor presentation (increase in suppression) was evident in homogeneous contexts. It could be because participants prepare for 
Heterogeneous

First

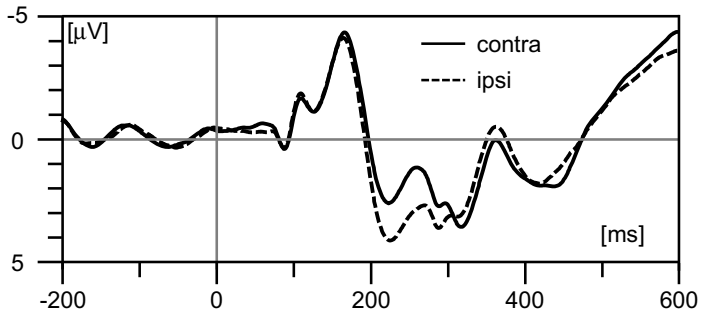

Second +

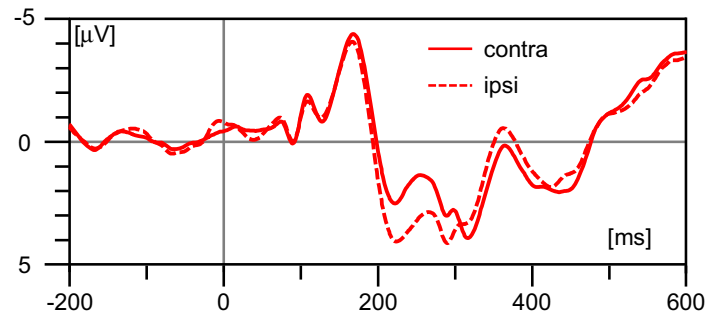

Difference

Waves (contra-ipsi)

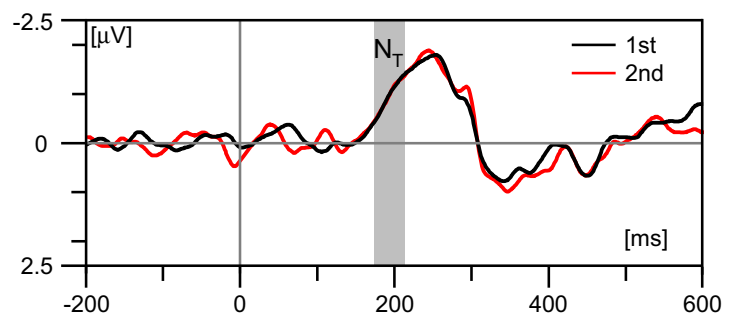

Homogenoeus
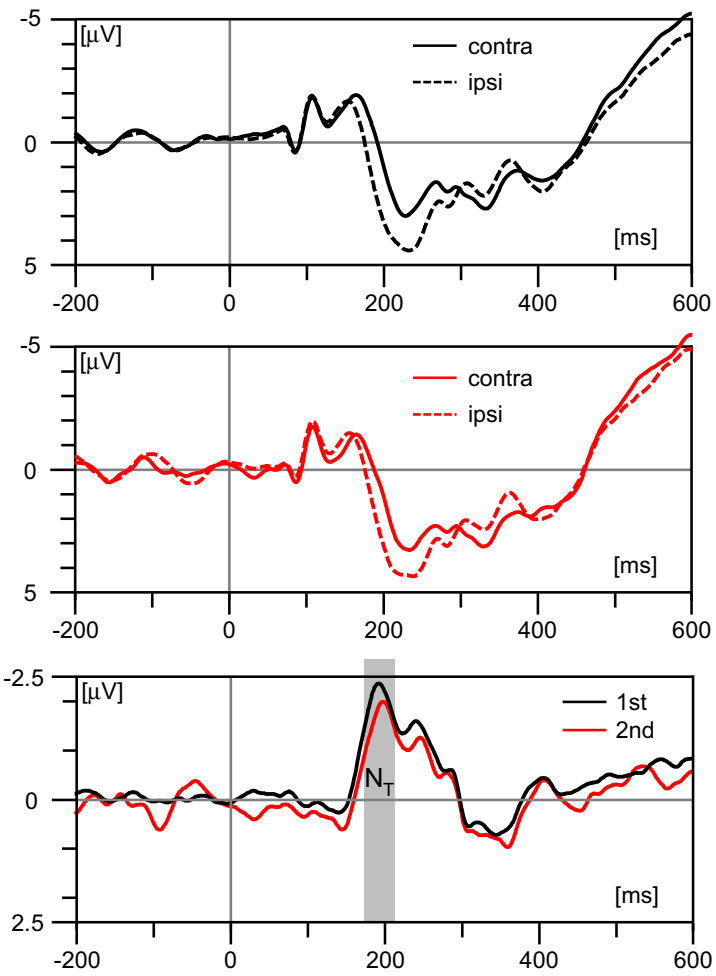

Fig. 6 Unpredictable order, targets lateral. Grand average ERPs recorded at posterior-occipital electrode sites (pool of PO3/4 and PO7/8), elicited by search displays for trials with heterogeneous contexts (left column) and trials with homogeneous contexts (right column) in trials with lateral targets in unpredictable blocks. The first row shows trials in which a distractor is presented the first time in a series. The second row shows data for second and more distractor color presentation. The bottom row shows the same data as in rows 1-2 but as difference waves (contra minus ipsi in relation to the target). Epochs for statistical analyses are shaded gray. For illustration purposes, EEG waveforms were low-pass filtered at $30 \mathrm{~Hz}$ (half power cutoff, $24 \mathrm{~dB}$ ) using digital filtering

defining dimension). An orientation-defined target is known to be less salient when presented in a context of heterogeneous distractor orientations (e.g., Duncan \& Humphreys, 1989) whereas the salience of a color-defined additional singleton supposedly remains the same. As a result, the target may appear less salient in heterogeneous than in homogeneous contexts, compared to the additional color singleton. Thus, the relatively more salient color distractor may "win the race" in heterogeneous contexts more likely than in homogeneous contexts (cf. Olivers \& Eimer, 2011). In addition, because the visual signal on which selective attention operates is noisy, a salient distractor may be more likely to accidentally be selected in heterogeneous contexts, potentially leading to attentional misguidance (Todd \& Kramer, 1994). In any case, the ratio of volitional control to bottom-up processing may also be modulated by physical properties of the visual field. We argue that because the physical properties varied between context types, top-down attention deployment was also modulated. As a result, when homogeneous contexts that offer more favorable physical properties were presented, visual search was less vulnerable to interference from distracting low-level features, and, eventually, observers had an easier time following their goal to respond to the target. 
The distractor-elicited $\mathrm{P}_{\mathrm{D}}$ component that is indicative of active suppression, was neither modulated by predictability nor by repetition. Apparently, suppression of irrelevant information was efficient regardless of the expectation or intertrial priming. The order of $\mathrm{N}_{D}$ and $\mathrm{P}_{\mathrm{D}}$ (a contralateral negativity followed by a contralateral positivity to the salient distractor) is in line with the notion that salient stimuli first capture attention before they can be actively suppressed (Sawaki \& Luck, 2010, 2013).

\section{Mechanisms of distractor-intertrial priming}

Clinical studies have suggested that intertrial priming through distractor repetition operates at a very early level of visual processing. For example, hemispatial neglect patients with intact occipital cortices and frontal eye fields show improved performance for repeated distractors, suggesting that intertrial priming is related to low-level visual processing rather than to higher level vision and attentional processing (Saevarsson et al., 2008; see also Finke et al., 2009). It was previously shown that intertrial priming of targets can be penetrated by top-down control (Fecteau, 2007; Hillstrom, 2000; Müller et al., 2004).We found that repetition of a specific distractor color only helps processing of a concurrent target if (1) the distractor color is expected and (2) if the context is heterogeneous. Thus, it appears that intertrial priming of distractors is modulated by top-down control as well; expectancy and context heterogeneity are both likely to modulate the ratio of topdown and bottom-up control. (1) When observers expect a particular feature to show up, they are more likely to take advantage of it and process it faster by more efficient topdown guidance; intertrial priming can be potentiated in cases in which target features were repeated and expected (Hillstrom, 2000; see also Leonard \& Egeth, 2008). (2) Analogously, heterogeneous contexts are more vulnerable to bottom-up interference than homogeneous contexts and hence increase the probability of attentional capture before volitional control can intervene (Feldmann-Wüstefeld \& Schubö, 2013) [footnote 1]. Thus, the present results suggest that, contrarily to Müller et al. (2004), intertrial priming at the feature level can also be subject to variations in top-down control.

According to the dimensional-weighting account (Found \& Müller, 1996; Müller et al., 2004), high weights for taskrelevant features and lower weights for task-irrelevant features can carry over to subsequent trials automatically, which may also account for intertrial priming (Becker et al., 2009). In the present study, we found evidence for such carry-over effects in both behavioral and EEG data. The second or third successive appearance of a distractor color decreased search speed (as indicated by RTs) and decreased attentional capture by the distractor (as indicated by $\mathrm{N}_{\mathrm{D}}$ ). In terms of dimensional weighting, a particular color can receive a low weight if a task-irrelevant items (here: the distractor) carries that feature.
This allows efficient processing of the target. If the distractor carries the same feature in the subsequent trial, it receives even lower weights (1) because of being a distractor and (2) because of the lingering low weight for that feature from the previous trial. As a consequence, the low weights of distractors allow even more efficient processing of the target because of less interference.

The present data also show that intertrial priming of distractors is only observed under certain circumstances. Search displays with homogeneous contexts showed no signs of intertrial priming of distractor features. This suggests that when contexts render visual search relatively robust against repeated distraction by salient stimuli, a repetition of the distracting feature does not further decrease attentional capture and thus does not further improve search performance. In line with this, it was previously found in an eye-tracking study that repeating an irrelevant distractor color did not alter saccade trajectories in a homogeneous context when color changes were unpredictable (Becker et al., 2014). Search displays with heterogeneous contexts, however, can benefit from repetition of an irrelevant distractor feature, but only if the color is systematically varying. This suggests that the observer's (implicit) knowledge about the upcoming repetition of a distractor moderates intertrial priming. Low weights for distractors only carry over to the next trial when a repetition is expected. This strongly suggests that intertrial priming is not only driven by bottom-up mechanisms but can also be affected by the volitional suppression of specific features or "history-driven sources of selection bias" (Awh et al., 2012, p. 442).

In line with this, Hillstrom (2000) found that intertrial priming for targets was more pronounced if targets were systematically varied than when they were randomly varied. Hillstrom argued that observers may use different strategies in blocks with systematic and random variation of the target feature: top-down-driven search in the systematic variation block, to make use of the preknowledge about relevant features; bottom-up-driven search in the random variation blocks, in which the upcoming relevant feature was unpredictable. A similar mechanism seems to be at work for distractordefining features: top-down-driven search allowed for making use of distractor feature repetition in blocks with systematic distractor color variation, whereas participants had to rely on a bottom-up-driven search in blocks with random distractor color variation. Such top-down-driven attentional weighting of target-defining features has previously been described for dimensions (Müller, Reimann, \& Krummenacher, 2003). The present results suggest that within dimensions, particular features can be weighted in a similar top-down manner.

Acknowledgments This research was supported by the Deutsche Forschungsgemeinschaft (German Research Foundation; SFB/TRR 135, TP B3). 


\section{References}

Awh, E., Belopolsky, A. V., \& Theeuwes, J. (2012). Top-down versus bottom-up attentional control: A failed theoretical dichotomy. Trends in Cognitive Sciences, 16(8), 437-443. doi:10.1016/j.tics. 2012.06.010

Becker, S. I. (2008). The mechanism of priming: Episodic retrieval or priming of pop-out? Acta Psychologica, 127(2), 324-339. doi:10. 1016/j.actpsy.2007.07.005

Becker, S. I., Ansorge, U., \& Horstmann, G. (2009). Can intertrial priming account for the similarity effect in visual search? Vision Research, 49(14), 1738-1756. doi:10.1016/j.visres.2009.04.001

Becker, S. I., Harris, A. M., Venini, D., \& Retell, J. D. (2014). Visual search for color and shape : When is the gaze guided by feature relationships, when by feature values? Journal of Experimental Psychology. Human Perception and Performance, 40(1), 264-291. doi: $10.1037 / \mathrm{a} 0033489$

Burra, N., \& Kerzel, D. (2013). Attentional capture during visual search is attenuated by target predictability: Evidence from the N2pc, Pd, and topographic segmentation. Psychophysiology, 50(5), 422-430. doi: 10.1111/psyp. 12019

Cousineau, D. (2005). Confidence intervals in within-subject designs : A simpler solution to Loftus and Masson's method. Tutorials in Quantitative Methods for Psychology, 1(1), 42-45.

Duncan, J., \& Humphreys, G. W. (1989). Visual search and stimulus similarity. Psychological Review, 96(3), 433-458. doi:10.1037// 0033-295X.96.3.433

Eimer, M., Kiss, M., \& Cheung, T. (2010). Priming of pop-out modulates attentional target selection in visual search: Behavioural and electrophysiological evidence. Vision Research, 50(14), 1353-1361. doi: 10.1016/j.visres.2009.11.001

Fecteau, J. H. (2007). Priming of pop-out depends upon the current goals of observers. Journal of Vision, 7(6), 1. doi:10.1167/7.6.1

Feldmann-Wüstefeld, T., Brandhofer, R., \& Schubö, A. (2016). Rewarded visual items capture attention only in heterogeneous contexts. Psychophysiology. doi:10.1111/psyp.12641

Feldmann-Wüstefeld, T., \& Schubö, A. (2013). Context homogeneity facilitates both distractor inhibition and target enhancement Anna Schub o. Journal of Vision, 13(3), 1-12. doi:10.1167/13.3.11

Finke, K., Bucher, L., Kerkhoff, G., Keller, I., Von Rosen, F., Geyer, T., ... Bublak, P. (2009). Inhibitory and facilitatory location priming in patients with left-sided visual hemi-neglect. Psychological Research, 73(2), 177-185. doi:10.1007/s00426-008-0209-8.

Folk, C. L., \& Remington, R. W. (2008). Bottom-up priming of top-down attentional control settings. Visual Cognition, 16(2-3), 215-231. doi:10.1080/13506280701458804

Found, A., \& Müller, H. J. (1996). Searching for unknown feature targets on more than one dimension: Investigating a "dimensionweighting" account. Perception \& Psychophysics, 58(1), 88-101. doi:10.3758/BF03205479

Geyer, T., Müller, H. J., \& Krummenacher, J. (2006). Cross-trial priming in visual search for singleton conjunction targets: Role of repeated target and distractor features. Perception \& Psychophysics, 68(5), 736-749. doi:10.3758/BF03193697

Hickey, C., Di Lollo, V., \& McDonald, J. (2009). Electrophysiological indices of target and distractor processing in visual search. Journal of Cognitive Neuroscience, 21(4), 760-775. doi:10.1162/jocn.2009. 21039

Hickey, C., Olivers, C., Meeter, M., \& Theeuwes, J. (2011). Feature priming and the capture of visual attention: Linking two ambiguity resolution hypotheses. Brain Research, 1370, 175-184. doi:10. 1016/j.brainres.2010.11.025

Hilimire, M. R., Hickey, C., \& Corballis, P. M. (2012). Target resolution in visual search involves the direct suppression of distractors:
Evidence from electrophysiology. Psychophysiology, 49(4), 504 509. doi:10.1111/j.1469-8986.2011.01326.x

Hillstrom, A. P. (2000). Repetition effects in visual search. Perception \& Psychophysics, 62(4), 800-817.

Kiss, M., Grubert, A., Petersen, A., \& Eimer, M. (2012). Attentional capture by salient distractors during visual search is determined by temporal task demands. Journal of Cognitive Neuroscience, 24(3), 749-759. doi:10.1162/jocn a 00127

Kristjánsson, Á., \& Campana, G. (2010). Where perception meets memory: A review of repetition priming in visual search tasks. Attention, Perception, \& Psychophysics, 72(1), 5-18. doi:10.3758/APP.72.1.5

Kristjánsson, Á., \& Driver, J. (2008). Priming in visual search: Separating the effects of target repetition, distractor repetition and role-reversal. Vision Research, 48(10), 1217-1232. doi:10.1016/j.visres.2008.02. 007

Kristjánsson, Á., Vuilleumier, P., Schwartz, S., Macaluso, E., \& Driver, J. (2007). Neural basis for priming of pop-out during visual search revealed with fMRI. Cerebral Cortex, 17(7), 1612-1624. doi:10. 1093/cercor/bhl072

Lamy, D., Antebi, C., Aviani, N., \& Carmel, T. (2008). Priming of popout provides reliable measures of target activation and distractor inhibition in selective attention. Vision Research, 48(1), 30-41. doi:10.1016/j.visres.2007.10.009

Lamy, D., Carmel, T., Egeth, H. E., \& Leber, A. B. (2006). Effects of search mode and intertrial priming on singleton search. Perception \& Psychophysics, 68(6), 919-932. doi:10.3758/BF03193355

Lamy, D., Yashar, A., \& Ruderman, L. (2013). Orientation search is mediated by distractor suppression: Evidence from priming of pop-out. Vision Research, 81, 29-35. doi:10.1016/j.visres.2013.01. 008

Leber, A. B., \& Egeth, H. E. (2006). Attention on autopilot: Past experience and attentional set. Visual Cognition, 14(4-8), 565-583. doi: $10.1080 / 13506280500193438$

Leonard, C. J., \& Egeth, H. E. (2008). Attentional guidance in singleton search : An examination of top-down, bottom-up, and intertrial factors. Visual Cognition, 16(January), 1078-1091. doi:10.1080/ 13506280701580698

Maljkovic, V., \& Nakayama, K. (1994). Priming of pop-out: I. Role of features. Memory \& Cognition, 22(6), 657-672. doi:10.3758/ BF03209251

Müller, H. J., Krummenacher, J., \& Heller, D. (2004). Dimension-specific intertrial facilitation in visual search for pop-out targets: Evidence for a top-down modulable visual short-term memory effect. Visual Cognition, 11(5), 577-602. doi:10.1080/13506280344000419

Müller, H. J., Reimann, B., \& Krummenacher, J. (2003). Visual search for singleton feature targets across dimensions: Stimulus- and expectancy-driven effects in dimensional weighting. Journal of Experimental Psychology: Human Perception and Performance, 29(5), 10211035. doi:10.1037/0096-1523.29.5.1021

Munneke, J., Fait, E., \& Mazza, V. (2013). Attentional processing of multiple targets and distractors. Psychophysiology, 50, 1104-1108. doi: $10.1111 /$ psyp. 12123

Olivers, C. N. L., \& Eimer, M. (2011). On the difference between working memory and attentional set. Neuropsychologia, 49(6), 15531558. doi:10.1016/j.neuropsychologia.2010.11.033

Saevarsson, S., Jóelsdóttir, S., Hjaltason, H., \& Kristjánsson, Á. (2008). Repetition of distractor sets improves visual search performance in hemispatial neglect. Neuropsychologia, 46(4), 1161-1169. doi:10. 1016/j.neuropsychologia.2007.10.020

Sawaki, R., Geng, J. J., \& Luck, S. J. (2012). A common neural mechanism for preventing and terminating the allocation of attention. Journal of Neuroscience, 32(31), 10725-10736. doi:10.1523/ JNEUROSCI.1864-12.2012

Sawaki, R., \& Luck, S. J. (2010). Capture versus suppression of attention by salient singletons: Electrophysiological evidence for an 
automatic attend-to-me signal. Attention, Perception, \& Psychophysics, 72(6), 1455-1470. doi:10.3758/APP.72.6.1455

Sawaki, R., \& Luck, S. J. (2013). Active suppression after involuntary capture of attention. Psychonomic Bulletin \& Review, 20(2), 296301. doi:10.3758/s13423-012-0353-4

Theeuwes, J. (2004). Top-down search strategies cannot override attentional capture. Psychonomic Bulletin \& Review, 11(1), 65-70. doi: 10.3758/BF03206462
Todd, S., \& Kramer, A. F. (1994). Attentional misguidance in visual search. Perception \& Psychophysics, 56(2), 198-210.

Wang, D., Kristjánsson, Á., \& Nakayama, K. (2005). Efficient visual search without top-down or bottom-up guidance. Perception \& Psychophysics, 67(2), 239-253. doi:10.3758/BF03206488

Woodman, G. F., \& Luck, S. J. (2003). Serial deployment of attention during visual search. Journal of Experimental Psychology: Human Perception and Performance, 29(1), 121-138. doi:10.1037/00961523.29.1.121 